\title{
Sliding mode control for altitude and attitude stabilization of quadrotor UAV with external disturbance
}

\author{
A. Noordin', M. A. M. Basri' ${ }^{2}$, Z. Mohamed ${ }^{3}$ \\ 1,2, ${ }^{3}$ School of Electrical Engineering, Universiti Teknologi Malaysia, Malaysia \\ ${ }^{1}$ Faculty of Electrical and Electronic Engineering Technology, Universiti Teknikal Malaysia Melaka, Malaysia
}

\begin{tabular}{l}
\hline Article Info \\
\hline Article historys: \\
Received Mar 26, 2019 \\
Revised May 3, 2019 \\
Accepted May 20, 2019 \\
\hline Keywords: \\
Distrubance \\
Quadrotor \\
Robustness \\
Saturation function \\
Sliding mode control
\end{tabular}

\begin{abstract}
This paper addresses the problem of robust altitude and attitude control of ' $x$ ' mode configuration quadrotor UAV using Lyapunov stability based sliding mode control with saturation function. The dynamic model of the quadrotor was derived by considering nonlinearity factor. MATLAB Simulink was used to simulate the model in two different conditions; without and with the presence of external disturbance. This was done to test the robustness of the control method. Simulation results showed that the sliding mode controller provides good performance and robustness against disturbance.
\end{abstract}

Copyright (C) 2019 Institute of Advanced Engineering and Science. All rights reserved.

\section{Corresponding Author:}

M. A. M. Basri,

School of Electrical Engineering, Universiti Teknologi Malaysia, 81310 Johor Bahru, Johor, Malaysia

Email: ariffanan@fke.utm.my

\section{INTRODUCTION}

Quadrotor as shown in Figure 1 has capabilities in vertical take-off and landing (VTOL), omnidirectional flying, and easy hovering performances in limited spaces. When compared to terrain mobile robot, quadrotor can cover mission at most range due to its capability to mobilize in environment with many obstacles. Furthermore, advancement in MEMS technology [1-4] and rapid prototyping technology had enabled the quadrotor to posses the simplest electronics and mechanical structural design. Therefore, quadrotor can always be considered for research with wide applications and various tasks such as civil, commercial, calamity, meteorological observation, agricultural, military related such as surveillances, reconnaissance and communication [5-7] as well as educational purposes [8].

As helicopter family, quadrotor can be controlled by varying the rotor speeds. For instance, to eliminate the anti-torque during manuaver, quadrotor configuration can be set up as followed; 1) two diagonal motors ( 1 and 2 ) are running in the same direction (counter clock wise) and 2) the other set of motors (3 and 4) in the other direction (clock wise) $[9,10]$.

However, quadrotor is an underactuated multi-input and multi-output system which has nonlinear dynamic behavior such as high coupling degree and unknown nonlinearities. These features make the control side of the quadrotor to be a challenging problem. Therefore, for a quadrotor to accomplish desired task, a robust capability of autonomous flight is required to maintain its stability in terms of attitude and navigation control [11]. Hence, most researchers encountered the greatest challenge in practical applications for flight controller design due to the very nonlinear quadrotor dynamics which were affected by the parametric uncertainties and external disturbances $[12,13]$. 
There has been many interest pertaining the use of sliding mode control for quadrotors and other VTOL vehicles. For example, [14] presented a controller for quadrotor that was split into two parts; 1) the sliding mode controller was applied to the attitude of the system, 2) a Proportional-Derivative controller was applied to the altitude and trajectory control of the system. Meanwhile, [15] compared between PD and SMC, [16] compared between PID and first order SMC, while [17] compared between second order SMC to controller developed in [10]. In [18], it applied a SMC driven by SMC disturbance observer for a small quadrotor vehicle. However, majority of the researchers used plus $(+)$ mode configuration model for quadrotor UAV.In this paper, a sliding mode control using saturation function were chose as it is known for its robustness against unmodeled dynamics, parametric uncertainties and external disturbances. We also consider the robust altitude and attitude tracking control of a quadrotor with $(\times)$ mode configuration with respect to external disturbance.

This paper is organised as follows. Section II describes the dynamic model of quadrotor in $(\times)$ mode configuration, design of the SMC for both attitude and altitude control of the quadrotor. Section III then presents simulation results of SMC controllers without and with the presence of external disturbance. Meanwhile, Section IV the conclusion of the paper.

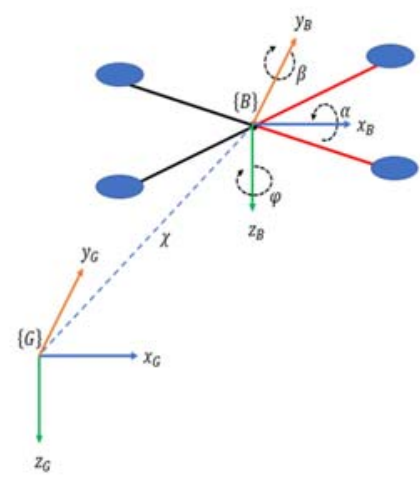

Figure 1. Inertial coordinate systems and body-fixed frame for $\times$-mode configuration quadrotor

\section{RESEARCH METHOD}

The following assumptions were used for modelling simplification [19].

a. The quadcopter was assumed as a rigid body.

b. The structure of quadrotor was assumed to be symmetrical with respect to the XY-axis.

c. The centre of mass and the origin of the body fixed frame are coinciding.

d. The propellers are considered as rigid and no blade flapping occurs.

e. The four propellers worked under the same conditions at any time, meaning that thrust coefficient, $b$ and reaction torque coefficient, $d$ are the same for all propellers.

Based on Figure 1, the relation of quadrotor body reference frame $\{B\}$, respected to earth reference frame $\{G\}$ must satisfy $\{B\}^{T}=R_{T} \times\{G\}^{T}$. Hence, the coordinate of quadrotor is described as followed:

$$
\begin{aligned}
& \chi=[x, y, z]^{T} \in \mathbb{R}^{3} \\
& \Upsilon=[\alpha, \beta, \varphi]^{T} \in \mathbb{R}^{3} \\
& R_{T}=\left[\begin{array}{ccc}
C \varphi C \beta & -S \varphi C \alpha+C \varphi S \beta S \alpha & S \varphi S \alpha+C \varphi S \beta C \alpha \\
S \varphi C \beta & C \varphi C \alpha+S \varphi S \beta S \alpha & -C \varphi S \alpha+S \varphi S \beta C \alpha \\
-S \beta & C \beta S \alpha & C \beta C \alpha
\end{array}\right]
\end{aligned}
$$

where, vector $\chi$ in (1) denotes the position of the quadrotor, while vector $\Upsilon$ in (2) denotes the attitude of the quadrotor. From (3) defines the rotation matrix $R_{T}$, where, $S$ and $C$ stands for trigonometric operators 'sine' and 'cosine' respectively. Table 1 Shows $\times$-mode configuration quadrotor's parameter used in this simulation [20].

Indo. J. Elec. Eng. \& Inf, Vol.7, No. 2, June 2019: 203 - 210 
The quadrotor dynamics in (4) and (5) are expressed using Newton-Euler translational and rotational dynamics formulation, where, $g$ is gravitational coefficient, $l$ is lateral arm length and $E_{z}$ vector matrix of $\mathrm{Z}$-axis is defined as $\left[\begin{array}{lll}0 & 0 & 1\end{array}\right]^{T} . I$ is the moments of inertia for the quadrotor, a diagonal matrix 3-by-3 and defined as $I=$ diagonal $\left[I_{x x} I_{y y} I_{z z}\right]^{T} . J_{r}$ is rotor inertia, $\Omega_{d}$ is total rotor speeds and lastly, $U_{1}, U_{2}, U_{3}$ and $U_{4}$ are used as control inputs.

$$
\begin{aligned}
& m \ddot{\chi}=m g E_{z}+U_{1} R_{T} E_{z} \\
& I \ddot{\Upsilon}=-\Upsilon \times I \Upsilon-J_{r}\left(\Upsilon \times E_{z}\right) \Omega_{d}+\left[U_{2} U_{3} U_{4}\right]^{T}
\end{aligned}
$$

Finally, by expanding (4) and (5), equation of translation dynamics and rotational dynamics for the quadrotor can be formulated as:

$$
\begin{aligned}
& \ddot{x}=\frac{U_{1}}{m}(S \varphi S \alpha+C \varphi S \beta C \alpha) \\
& y=\frac{U_{1}}{m}(-C \varphi S \alpha+S \varphi S \beta C \alpha) \\
& \ddot{z}=g+\frac{U_{1}}{m}(C \beta C \alpha) \\
& \ddot{\alpha}=\left(\frac{I_{y y}-I_{z z}}{I_{x x}}\right) \dot{\varphi} \dot{\beta}-\left(\frac{J_{r} \Omega_{d}}{I_{x x}}\right) \dot{\beta}+\left(\frac{l}{I_{x x}}\right) U_{2} \\
& \ddot{\beta}=\left(\frac{I_{z z}-I_{x x}}{I_{y y}}\right) \dot{\varphi} \dot{\alpha}+\left(\frac{J_{r} \Omega_{d}}{I_{y y}}\right) \dot{\alpha}+\left(\frac{l}{I_{y y}}\right) U_{3} \\
& \ddot{\varphi}=\left(\frac{I_{x x}-I_{y y}}{I_{z z}}\right) \dot{\beta} \dot{\alpha}+\left(\frac{1}{I_{z z}}\right) U_{4}
\end{aligned}
$$

with thrust coefficient, $b$ and drag coefficient, $d$, the inputs are mapped by:

$$
\begin{aligned}
& U_{1}=b\left(\Omega_{1}^{2}+\Omega_{2}^{2}+\Omega_{3}^{2}+\Omega_{4}^{2}\right) \\
& U_{2}=\frac{b}{\sqrt{2}}\left(-\Omega_{1}^{2}+\Omega_{2}^{2}-\Omega_{3}^{2}+\Omega_{4}^{2}\right) \\
& U_{3}=\frac{b}{\sqrt{2}}\left(-\Omega_{1}^{2}+\Omega_{2}^{2}+\Omega_{3}^{2}-\Omega_{4}^{2}\right) \\
& U_{4}=d\left(\Omega_{1}^{2}+\Omega_{2}^{2}-\Omega_{3}^{2}-\Omega_{4}^{2}\right)
\end{aligned}
$$

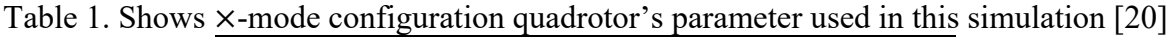

\begin{tabular}{cccc}
\hline Specification & Parameter & Unit & Value \\
\hline Quadrotor mass & $m$ & $\mathrm{~kg}$ & 1.033 \\
Lateral moment arm & $l$ & $\mathrm{~m}$ & 0.225 \\
Thrust coefficient & $b$ & $\mathrm{Ns}^{2}$ & $2.8625 \times 10-7$ \\
Drag coefficient & $d$ & $\mathrm{Nms}^{2}$ & $4.4212 \times 10-10$ \\
Rolling moment of inertia & $I_{x x}$ & $\mathrm{kgm}^{2}$ & 0.0183 \\
Pitching moment of inertia & $I_{y y}$ & $\mathrm{kgm}^{2}$ & 0.0183 \\
Yawing moment of inertia & $I_{z z}$ & $\mathrm{kgm}^{2}$ & 0.0385 \\
\hline
\end{tabular}

\subsection{Controller design}

The dynamic model in (6) and (7) can be represented into nonlinear dynamic equation described as:

$$
\ddot{X}=f(X)+g(X) U
$$

where $U=\left[\begin{array}{llll}U_{1} & U_{2} & U_{3} & U_{4}\end{array}\right]^{T}$, and $X=\left[\begin{array}{lllll}x & y & z & \alpha & \beta\end{array}\right]^{T}$ were the input, and state respectively. From (6) and (7), the nonlinear dynamic function $f(X)$ and nonlinear control function $g(X)$ matrices can be written accordingly as:

$$
f(X)=\left(\begin{array}{c}
0 \\
0 \\
g \\
a_{1} \dot{\varphi} \dot{\beta}+a_{2} \Omega_{d} \dot{\beta} \\
a_{3} \dot{\varphi} \dot{\alpha}+a_{4} \Omega_{d} \dot{\alpha} \\
a_{5} \dot{\beta} \dot{\alpha}
\end{array}\right)
$$




$$
g(X)=\left(\begin{array}{cccc}
u_{x} \frac{1}{m} & 0 & 0 & 0 \\
u_{y} \frac{1}{m} & 0 & 0 & 0 \\
u_{z} \frac{1}{m} & 0 & 0 & 0 \\
0 & b_{1} & 0 & 0 \\
0 & 0 & b_{2} & 0 \\
0 & 0 & 0 & b_{3}
\end{array}\right)
$$

where $a_{1}=\frac{I_{y y}-I_{z z}}{I_{x x}}, a_{2}=-\frac{J_{r}}{I_{x x}}, a_{3}=\frac{I_{z z}-I_{x x}}{I_{y y}}, a_{4}=\frac{J_{r}}{I_{y y}}, a_{5}=\frac{I_{x x}-I_{y y}}{I_{z z}}, b_{1}=\frac{l}{I_{x x}}, b_{2}=\frac{l}{I_{y y}}$ and $b_{3}=\frac{1}{I_{z z}}$. A sliding surface is defined as:

$$
s=\dot{e}+\lambda e
$$
is defined as:

Commonly, $\dot{s}$ will be represented as $\dot{s}=-k \operatorname{sgn}(s)$ where $k$ is positive constant and signum function

$$
\operatorname{sgn}(s)= \begin{cases}+1 & s>0 \\ 0 & s=0 \\ -1 & s<0\end{cases}
$$

In practice, switching control law, needs high frequency to switch across the surface which caused oscillation within switching surface. This effect is called chattering phenomenon as shown in Figure 2.

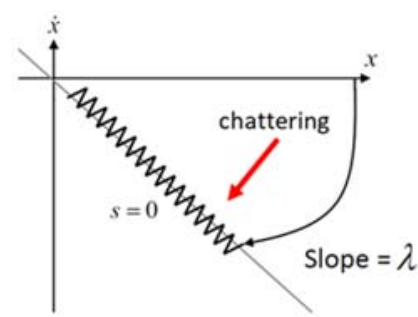

Figure 2. Chattering phenomenon on sliding surface

In order to reduce the chattering effects, a boundary method is applied by replacing the signum function with saturation function. The saturation function was defined as (13), where boundary layer was bounded by $0<\phi_{b}<1$. Figure 3 illustrates the saturation function in sliding surface.

$$
\operatorname{sat}(s)\left\{\begin{array}{cr}
-1 & s<-\phi_{b} \\
\frac{s}{\phi_{b}} & -\phi_{b} \leq s \leq \phi_{b} \\
1 & s>\phi_{b}
\end{array}\right.
$$

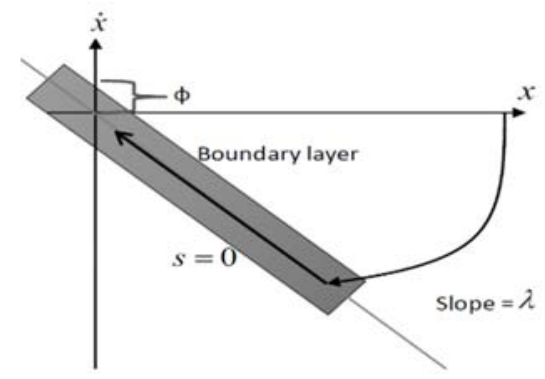

Figure 3. Boundary layer to minimize chattering phenomenon 


\subsection{Altitude control}

By making $\dot{s}=-k \operatorname{sat}\left(\frac{S}{\phi_{b}}\right)$, where $k$ is the coefficient of exponential approach law and must be greater than zero, $k>0$. The corresponding control law is designed as:

$\dot{s}=\left(\ddot{z}_{d}-\ddot{z}\right)+\lambda_{z}\left(\dot{z}_{d}-\dot{z}\right)$

Rearrange (6) and (15) for the control law gives:

$$
U_{1}=\frac{m}{u_{z}}\left(\ddot{z}_{d}-g+\lambda_{z}\left(\dot{z}_{d}-\dot{z}\right)+k_{z} \operatorname{sat}\left(\frac{s}{\phi_{b}}\right)\right)
$$

\subsection{Attitude control}

In this section, same technique for guaranteed stability was designed using error tracking and same sliding manifolds defined as:

$$
\begin{aligned}
& e_{\alpha}=\alpha_{d}-\alpha \\
& e_{\beta}=\beta_{d}-\beta \\
& e_{\varphi}=\varphi_{d}-\varphi \\
& s_{\alpha}=\dot{e}_{\alpha}+\lambda_{\alpha} e_{\alpha} \\
& s_{\beta}=\dot{e}_{\beta}+\lambda_{\beta} e_{\beta} \\
& s_{\varphi}=\dot{e}_{\varphi}+\lambda_{\varphi} e_{\varphi}
\end{aligned}
$$

The control input can then be obtained after (7), (17) and (18) are rearranged and described as:

$$
\begin{aligned}
& U_{2}=\frac{1}{b_{1}}\left(\ddot{\alpha}_{d}-a_{1} \dot{\varphi} \dot{\beta}-\mathrm{a}_{2} \dot{\beta}+\lambda_{\alpha}\left(\dot{\alpha}_{d}-\dot{\alpha}\right)+k_{\alpha} \operatorname{sat}\left(\frac{s_{\alpha}}{\phi_{b}}\right)\right) \\
& U_{3}=\frac{1}{b_{2}}\left(\ddot{\beta}_{d}-a_{3} \dot{\varphi} \dot{\alpha}-a_{4} \Omega_{d} \dot{\alpha}+\lambda_{\beta}\left(\dot{\beta}_{d}-\dot{\beta}\right)+k_{\beta} \operatorname{sat}\left(\frac{s_{\beta}}{\phi_{b}}\right)\right) \\
& U_{4}=\frac{1}{b_{3}}\left(\ddot{\varphi}_{d}-a_{5} \dot{\beta} \dot{\alpha}+\lambda_{\varphi}\left(\dot{\varphi}_{d}-\dot{\varphi}\right)+k_{\varphi} \operatorname{sat}\left(\frac{s_{\varphi}}{\phi_{b}}\right)\right)
\end{aligned}
$$

\subsection{Stability}

Lyapunov function can be assumed as:

$V\left(s_{i}\right)=\frac{1}{2} s_{i}^{2}(i=z, \alpha, \beta, \varphi)$

Derivation of (20) gives:

$\dot{V}\left(s_{i}\right)=s_{i} \dot{s}_{i}$

Replacing $\dot{S}_{i}=-k \operatorname{sat}\left(\frac{s_{i}}{\phi_{b}}\right)$ into $(21)$ gives:

$$
\dot{V}\left(s_{i}\right)=s_{i} \dot{s}_{i}=-s_{i} k s a t\left(\frac{s_{i}}{\phi_{b}}\right)<0
$$

Therefore, if $\dot{V}\left(s_{i}\right)<0$, so $s_{i} \dot{s}_{i}<0$ the sliding condition will be verified and guaranteed by Lyapunov stability.

\section{RESULT AND ANALYSIS}

A control using sliding mode with saturation function switching control law were simulated for altitude and attitude control of quadrotor UAV. Figure 4 shows the result of altitude and attitude control where the initial altitude $z$, is set to zero and the desired height is set at 10 meters. Whereas initial attitude,

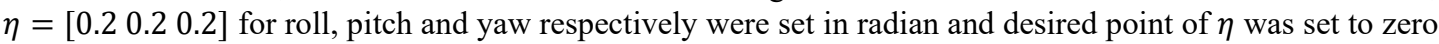
attitude stabilization. 

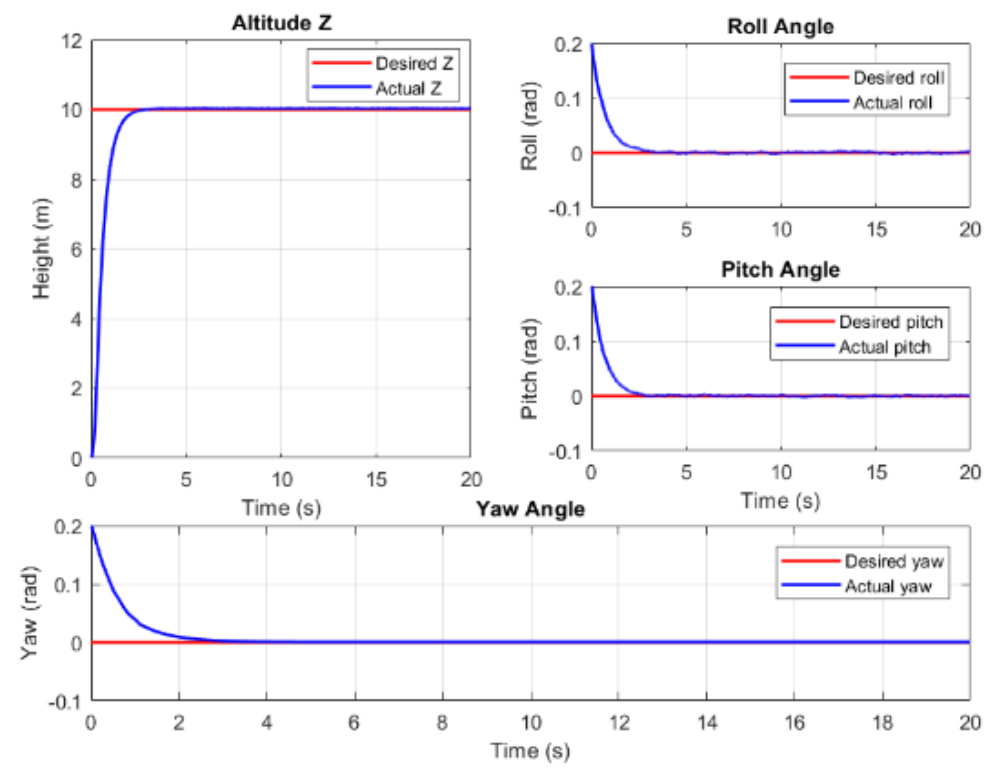

Figure 4. Altitude and attitude control without disturbances

The simulation was then performed with the model external disturbances. The model in (9) was augmented to:

$$
\ddot{X}=f(X)+g(X) U+d
$$

Where $d$ is unknown external disturbance vector. For this simulation the external disturbance, $d$ is taken as normal gaussian noise [15]:

$$
d=[\mathcal{N}(0,0.5) \mathcal{N}(0,0.5) \mathcal{N}(0,0.5) \mathcal{N}(0,0.5)]
$$

Figure 5 shows the performance of quadrotor UAV with SMC controller with external disturbances in the form of normal gaussian noise. It indicates that with the appearance of disturbance, SMC controller with saturation function still give fast response with slight amount of oscillation. However, the control inputs were sensitive to the noise especially at $U_{1}$ after 2.5 seconds period as shown in Figure 6.
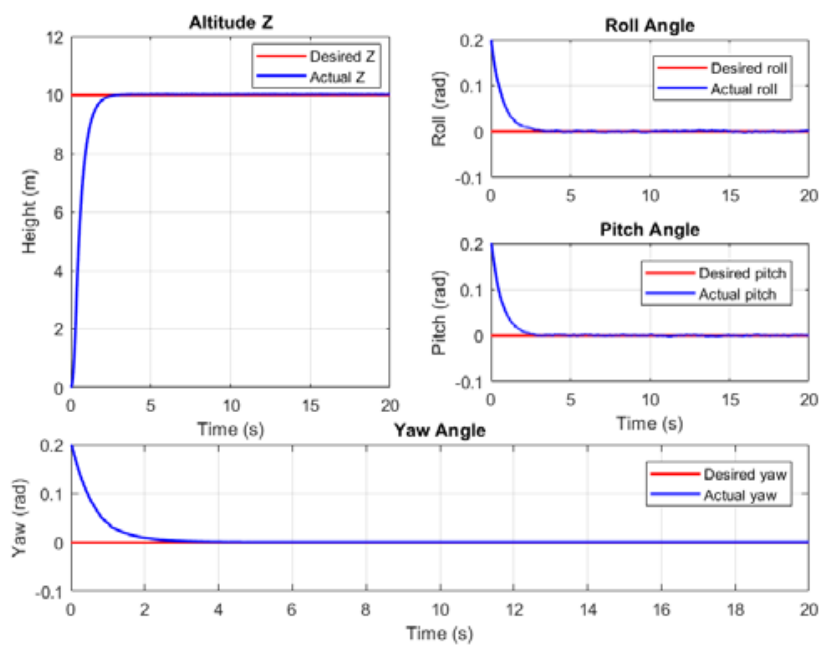

Figure 5. Altitude and attitude control with the presence of disturances

Indo. J. Elec. Eng. \& Inf, Vol.7, No. 2, June 2019: 203 - 210 

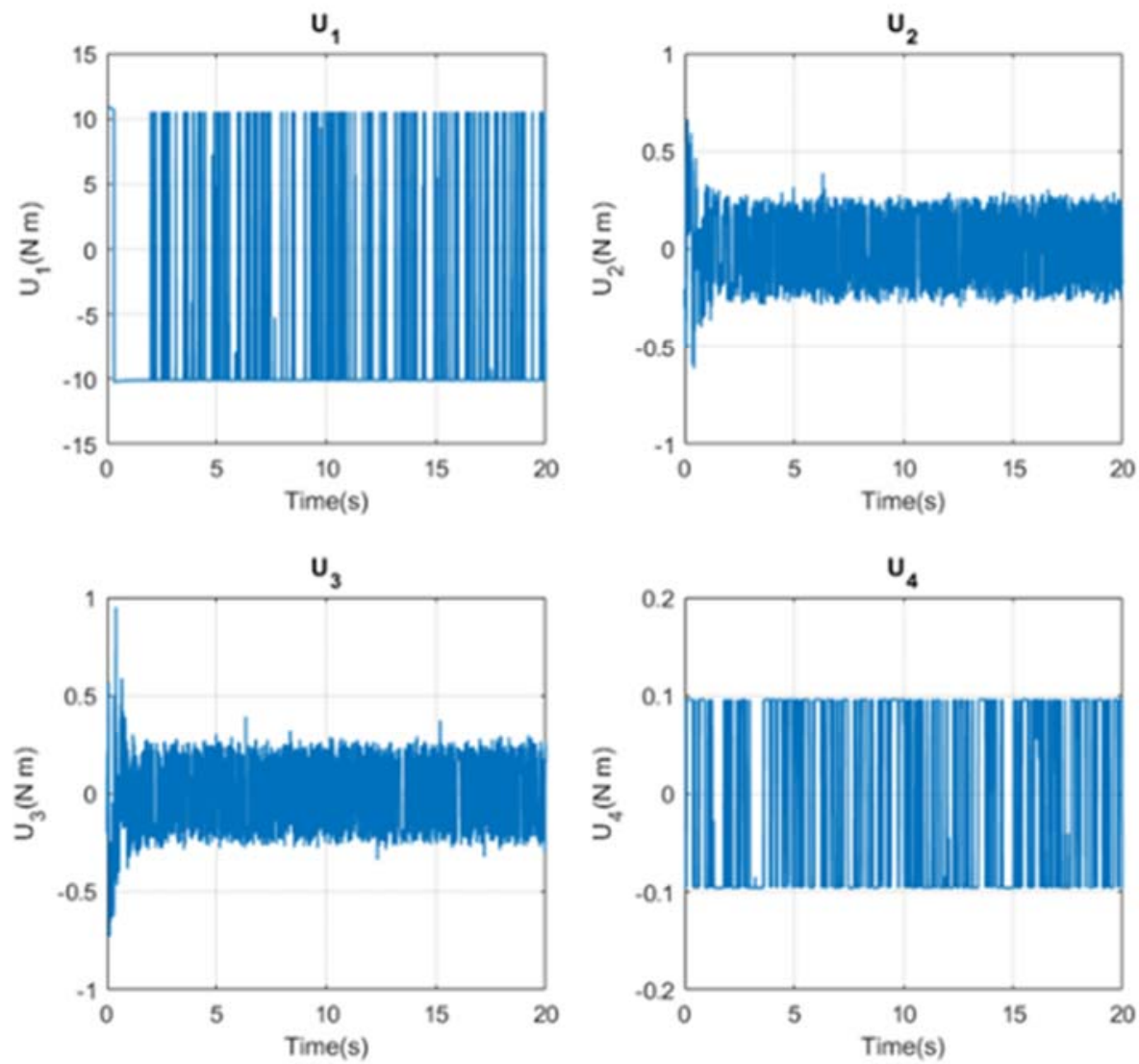

Figure 6. Sliding mode control inputs with the presence of disturbances

The performance of the proposed method was compared with a method published in [21]. As compared to a discrete PID and nonlinear model predictive Control (MPC) that had been implemented in [21], the simulation results showed that the quadrotor UAV can be stabilized at the desired altitude and attitude with minimum chattering problem in control effort. In robustness test (presence of external disturbances), [21] used uniform random noise while in this paper used normal gaussian noise. Both discrete PID and MPC in [21] and SMC in this paper have shown good performance of quadrotor. However, SMC shows better response without overhoot or undershoot compared to [21].

\section{CONCLUSION}

This paper present altitude and attitude control using nonlinear sliding mode control using saturation function. The simulation results demonstrate the quadrotor UAV can stabilize at the desired altitude and attitude with minimum chattering problem in control effort. In the test with presence of external disturbances, SMC controller performs well. For a future work, the sliding mode using Gao's power rate reaching law and tanh function can be simulated to see the robustness of the control technics while reducing the chattering phenomenon.

\section{ACKNOWLEDGEMENTS}

The authors would like to thank Universiti Teknologi Malaysia (UTM) under the Research University Grant (Q.J130000.2523.15H39), Universiti Teknikal Malaysia Melaka (UTeM), and Ministry of Education Malaysia for supporting this research. 


\section{REFERENCES}

[1] J. Chang, J. Cieslak, A. Zolghadri, J. Dávila, and J. Zhou, "Quadrotor attitude estimation with gyroscope bias reconstruction capabilities," IFAC-PapersOnLine, vol. 49, no. 5, pp. 260-265, 2016.

[2] V. Kriz and P. Gabrlik, "UranusLink-Communication protocol for UAV with small overhead and encryption ability," in IFAC Proceedings Volumes (IFAC-PapersOnline), vol. 48, no. 4, pp. 474-479, 2015.

[3] P. J. Bristeau, F. Callou, D. Vissière, and N. Petit, "The Navigation and Control technology inside the AR.Drone micro UAV," in IFAC Proceedings Volumes (IFAC-PapersOnline), vol. 18, no. PART 1, pp. 1477-1484, 2011.

[4] H. Jafari, M. Zareh, J. Roshanian, and A. Nikkhah, "An Optimal Guidance Law Applied to Quadrotor Using LQR Method," Trans. Jpn. Soc. Aeronaut. Space Sci., vol. 53, no. 179, pp. 32-39, 2010.

[5] J. Ajmera and V. Sankaranarayanan, "Point-to-point control of a quadrotor: Theory and experiment," in IFACInternational Federation of Automatic Control, vol. 49, no. 1, pp. 401-406, 2016.

[6] J. Bazin, T. Fields, and A. J. Smith, "Feasibility of In-Flight Quadrotor Individual Motor Thrust Measurements," AIAA Atmos. Flight Mech. Conf., no. January, pp. 1-12, 2016.

[7] D. B B V L and P. Singh, "A survey on design and development of an unmanned aerial vehicle (quadcopter)," Int. J. Intell. Unmanned Syst., vol. 4, no. 2, pp. 70-106, 2016.

[8] J. Števek and M. Fikar, "Teaching Aids for Laboratory Experiments with AR.Drone2 Quadrotor," IFACPapersOnLine, vol. 49, no. 6, pp. 236-241, 2016.

[9] D. Domingos, G. Camargo, and F. Gomide, "Autonomous Fuzzy Control and Navigation of Quadcopters," IFACPapersOnLine, vol. 49, no. 5, pp. 73-78, 2016.

[10] S. Bouabdallah, P. Murrieri, R. Siegwart, B. Ghder, and P. R. Bud, "Design and Control of an Indoor Micro Quadrotor," Proc. 2004 IEEE Int. Conf. Robot. 8 Autom. New Orleans, LA April 2004, pp. 4393-4398, 2004.

[11] A. T. Gaitan and Y. Bolea, "Modeling and Robust Attitude Control of a Quadrotor System," 10th Int. Conf. Electr. Eng. Comput. Sci. Autom. Control, pp. 7-12, 2013.

[12] Y. Bai, H. Liu, Z. Shi, and Y. Zhong, "Robust Control of Quadrotor Unmanned Air Vehicles," Proc. - 201231 st Chinese Control Conf., pp. 4462-4467, 2012.

[13] L. I. U. Hao, T. Yu, and Y. U. Yao, "Robust Trajectory Tracking Control for Quadrotors with Uncertainties and Delays," Proc. 35th Chinese Control Conf., pp. 2998-3001, 2016.

[14] K. Runcharoon and V. Srichatrapimuk, "Sliding Mode Control of Quadrotor," 2013 Int. Conf. Technol. Adv. Electr. Electron. Comput. Eng., no. 1, pp. 552-557, 2013.

[15] M. K. Shaik and J. F. Whidborne, "Robust Sliding Mode Control of a Quadrotor," UKACC 11th Int. Conf. Control, pp. 1-6, 2016.

[16] P. Dey, "Robust Attitude Control of Quadrotor using Sliding Mode," Int. Conf. Autom. Control Dyn. Optim. Tech., pp. 268-272, 2016.

[17] L. Luque-Vega, B. Castillo-Toledo, and A. G. Loukianov, "Robust block second order sliding mode control for a quadrotor," J. Franklin Inst., vol. 349, no. 2, pp. 719-739, 2012.

[18] L. Besnard, Y. B. Shtessel, and B. Landrum, "Control of a Quadrotor Vehicle Using Sliding Mode Disturbance Observer," 2007 Am. Control Conf., pp. 5230-5235, 2007.

[19] M. A. Mohd Basri, A. R. Husain, and K. A. Danapalasingam, "Enhanced Backstepping Controller Design with Application to Autonomous Quadrotor Unmanned Aerial Vehicle," J. Intell. Robot. Syst. Theory Appl., vol. 79, no. 2, pp. 295-321, 2014.

[20] A. Noordin, M. A. M. Basri, Z. Mohamed, and A. F. Z. Abidin, "Modelling and PSO fine-tuned PID control of quadrotor UAV," Int. J. Adv. Sci. Eng. Inf. Technol., vol. 7, no. 4, 2017.

[21] H. S. Khan and M. B. Kadri, "Attitude and altitude control of quadrotor by discrete PID control and non-linear model predictive control,” 2015 Int. Conf. Inf. Commun. Technol., pp. 1-11, 2015.

Indo. J. Elec. Eng. \& Inf, Vol.7, No. 2, June 2019: 203 - 210 